\title{
Mechanisms of after-hyperpolarization following activation of fly visual motion-sensitive neurons
}

\author{
Rafael Kurtz, Ulrich Beckers, Benjamin Hundsdörfer and Martin Egelhaaf \\ Department of Neurobiology, Bielefeld University, Postfach 1001 31, 33501 Bielefeld, Germany
}

Keywords: adaptation, calcium, invertebrate, vision, voltage clamp

\begin{abstract}
In many neurons, strong excitatory stimulation causes an after-hyperpolarization (AHP) at stimulus offset, which might give rise to activity-dependent adaptation. Graded-potential visual motion-sensitive neurons of the fly Calliphora vicina respond with depolarization and hyperpolarization during motion in their preferred direction and their anti-preferred direction, respectively. A prominent after-response, opposite in sign to the response during motion, is selectively expressed after stimulation with preferreddirection motion. Previous findings suggested that this AHP is generated in the motion-sensitive neurons themselves rather than in presynaptic processing layers. However, it remained unknown whether the AHP is caused by membrane depolarization itself or by another process, e.g. a signaling cascade triggered by activity of excitatory input channels. Here we showed by current injections and voltage clamp that the AHP and a corresponding current are generated directly by depolarization. To test whether the generation of an AHP is linked to depolarization via a $\mathrm{Ca}^{2+}$-dependent mechanism, we used photoactivation of a high-affinity $\mathrm{Ca}^{2+}$ buffer. In accordance with previous findings the AHP was insensitive to manipulation of cytosolic $\mathrm{Ca}^{2+}$. We propose that membrane depolarization presents a more direction-selective mechanism for the control of AHP than other potential control parameters.
\end{abstract}

\section{Introduction}

Sensory cells and neurons are often confronted with the problem of encoding an enormous range of possible input intensities. The available number of output states that can be faithfully discriminated from each other is, however, constrained by the presence of noise and saturation limits. One way to cope with this problem is activitydependent adaptation, in which an estimate of the current input strength based on stimulus history is used to control compression or a downward shift of the neuronal response (Laughlin, 1981; for review see Kohn, 2007). How this estimate is generated depends on the implemented cellular adaptation mechanism. For example, neurotransmitter receptors can express use-dependent plasticity and downregulate their sensitivity during periods of high input activity (for reviews see Hawkins et al., 2006; Bellone et al., 2008). However, depolarization of the cell can itself regulate the adaptation state (see e.g. Torkkeli et al., 2001; Vähäsöyrinki et al., 2006). Under certain circumstances adaptation expresses itself as an after-hyperpolarization (AHP) following strong excitatory stimulation (see e.g. Sanchez-Vives et al., 2000; Peron \& Gabbiani, 2009).

In the fly visual system, motion is first computed on a local basis from the time-varying visual input (for reviews see Egelhaaf, 2006, 2008). Tangential cells (TCs) integrate outputs of numerous motionsensitive local elements on their large dendrites and therefore have large receptive fields (for reviews see Hausen, 1984; Hausen \& Egelhaaf,

Correspondence: Dr R. Kurtz, as above.

E-mail: rafael.kurtz@uni-bielefeld.de

Received 24 March 2009, revised 2 June 2009, accepted 18 June 2009
1989; Egelhaaf et al., 2002; Borst \& Haag, 2002). About 60 TCs can be individually identified in the lobula plate of each brain hemisphere and are thought to provide major input to the motor system (Strausfeld \& Bassemir, 1985; Haag et al., 2007; Huston \& Krapp, 2008).

In fly TCs various adaptation mechanisms have been described, which differ in temporal characteristics and direction selectivity (Maddess \& Laughlin, 1985; Brenner et al., 2000; Harris et al., 2000; Kurtz et al., 2000; Neri, 2007). In the types of TCs analysed in the present study, the synaptic output consists of graded depolarizations and hyperpolarizations during motion in the preferred and antipreferred direction, respectively. Depolarization during motion in the preferred direction is followed by a prominent AHP. In contrast, motion in the anti-preferred direction elicits strong hyperpolarization but is not followed by a prominent after-response, either depolarizing or hyperpolarizing (Harris et al., 2000; Kurtz et al., 2000). Thus, AHP can be regarded as a consequence of direction-selective adaptation in TCs because the underlying cellular mechanism is activated during sustained motion in the preferred direction and brings the graded response closer to resting level.

The AHP of TCs is accompanied by a decrease in neuronal input resistance (Kurtz, 2007), suggesting that it is caused by a conductance that is activated when the neuron receives strong excitatory input. However, as in the previous studies visual stimulation was used to activate TCs, it was not possible to differentiate between several plausible cellular mechanisms underlying AHP. In this study we show directly by current injection and voltage clamp that membrane depolarization alone is sufficient to cause AHP, and we re-examine a potential role of $\mathrm{Ca}^{2+}$ in the control of AHP. 


\section{Materials and methods}

\section{Preparation and electrophysiology}

All experiments were carried out at room temperature $\left(18-25^{\circ} \mathrm{C}\right)$ on $=3$-day-old female blowflies, bred in the Department of Neurobiology, Bielefeld University. After dissection as described in Dürr \& Egelhaaf (1999), the fly was mounted under an upright fixed-stage microscope (Axioskop FS, Zeiss) or in front of a stereomicroscope to view the fly brain from behind. Membrane potential recordings, $\mathrm{Ca}^{2+}$ imaging and ultraviolet (UV) photolysis of caged $\mathrm{Ca}^{2+}$ buffer were performed in vivo on TCs in the third visual neuropile of the fly, the lobula plate. Identification of individual TCs was based on their receptive field properties, specific characteristics of their electrical responses and their anatomy, if visualized with fluorescent $\mathrm{Ca}^{2+}$ dyes. Three types of TCs were examined. The neurons of the 'horizontal system' (HS) and the 'vertical system' (VS) produce graded axonal depolarizations and hyperpolarizations in response to motion in the preferred and anti-preferred direction, respectively. Action potentials of variable amplitudes, so-called 'spikelets', are superimposed on the graded signal, in particular during preferred-direction motion (Hengstenberg, 1977, 1982; Hausen, 1982). The third type of TC, the purely graded 'centrifugal horizontal' $(\mathrm{CH})$ neuron (see Eckert \& Dvorak, 1983), receives input from HS neurons via dendro-dendritic electrical synapses (Haag \& Borst, 2002) and functions as an inhibitory element in a circuit for figure-ground discrimination (Warzecha et al., 2003). HS and VS cells are very similar in their biophysical properties (Borst \& Haag, 1996; Haag et al., 1997). We therefore do not regard it as critical that experiments on one or the other cell class contribute differentially to the different parts of the present study. Results from experiments on $\mathrm{CH}$ cells, which differ from VS and HS cells in their biophysical properties, are only shown in addition to results obtained with one of the other cell classes.

Intracellular recordings from TCs were made using sharp borosilicate glass electrodes (GC100TF-10; Clark Electromedical, Edenbridge, UK) pulled on a Brown-Flaming Puller (P-97; Sutter Instruments, San Rafael, CA, USA). The electrode resistance was $15-40 \mathrm{M} \Omega$ when filled with $1 \mathrm{M} \mathrm{KCl}$ or $2 \mathrm{M}$ potassium acetate and 30-80 M $\Omega$ when the electrode tip contained $\mathrm{Ca}^{2+}$ dye and caged $\mathrm{Ca}^{2+}$ (see below). HS and VS neurons were recorded from their axons close to their output sites. In $\mathrm{CH}$ neurons an axon in a strict sense is missing because the major dendrite in the lobula plate itself forms an extended output site. $\mathrm{CH}$ neurons were recorded from the long main neurite (termed 'axon' in some studies) connecting the major dendrite with the smaller dendritic ramification in the protocerebrum. Electrode signals for voltage recordings in bridged mode and current injections were amplified with an Axoclamp 2A (Axon Instruments, Foster City, CA, USA) and sampled at rates of $3 \mathrm{kHz}$ with an amplitude resolution of $0.0244 \mathrm{mV}$ by an analog-to-digital converter (DT2801A; Data Translation, Marlboro, MA, USA). For voltage-clamp recordings an SEC-10LX amplifier (npi electronics, Tamm, Germany) with the standard low-voltage headstage was used. These recordings were performed using the discontinuous single-electrode voltage-clamp method with a duty cycle of $1 / 4$ and a switching frequency of about $40 \mathrm{kHz}$. The waveform of the amplifier's discontinuous raw output signal was checked during each recording to tune the amplifier's filtering and gain properties to the electrode properties. In particular, care was taken that electrodes were fast enough, i.e. that during the amplifier's voltage sampling intervals the electrode voltage had recovered from the amplifier's current pulses to a quasi steady-state that is determined exclusively by the neuronal properties. In voltageclamp experiments the membrane potential and applied voltage-clamp current were recorded with a high-precision data acquisition board
(PD2-MFS-4-500/14; United Electronic Industries, Canton, MA, USA) at a sampling frequency of $100 \mathrm{kHz}$ for each channel.

\section{Ultraviolet photolysis of caged $\mathrm{Ca}^{2+}$ buffer and $\mathrm{Ca}^{2+}$ imaging}

The photolysis of caged $\mathrm{Ca}^{2+}$ buffer Diazo- 2 and $\mathrm{Ca}^{2+}$ imaging followed Kurtz (2004), apart from some minor modifications as detailed below. Caged $\mathrm{Ca}^{2+}$ buffer and fluorescent $\mathrm{Ca}^{2+}$ dye were injected from the electrode tip into single TCs during intracellular recording by applying 1-3 nA hyperpolarizing current for 5-10 min and left to diffuse throughout the cytoplasm for $=5 \mathrm{~min}$. The electrode tips contained $33 \mathrm{~mm}$ HEPES, $33 \mathrm{~mm} \mathrm{KCl,} 2 \mathrm{~mm} \mathrm{KOH,} 200 \mathrm{~mm}$ Diazo-2 tetrapotassium salt and $10 \mathrm{~mm}$ Oregon-green 488 1,2-bis(o-aminophenoxy)ethane-N,N, $\mathrm{N}^{\prime}, \mathrm{N}^{\prime}$-tetraacetic acid (BAPTA)-1 hexapotassium salt (fluorescence compounds from Molecular Probes, Eugene, OR, USA). With this procedure, step-like decreases of the cytosolic $\mathrm{Ca}^{2+}$ concentration, indicating rapid increases in $\mathrm{Ca}^{2+}$ buffer capacity, could be induced repetitively by delivering filtered light $(\lambda<360 \mathrm{~nm}$ or $<380 \mathrm{~nm}$ ) from a Xenon flash lamp (JML-C2; Rapp Optoelectronics, Hamburg, Germany) via a quartz light guide (diameter $200 \mu \mathrm{m}$ ). Due to the sensitivity to UV light of the fly's photoreceptors (Kirschfeld \& Franceschini, 1977), visual responses are observed in TCs during UV photolysis of caged compounds (Kurtz, 2004). We applied two types of controls to distinguish potential cellular responses that are elicited by an increased concentration of cytosolic $\mathrm{Ca}^{2+}$ buffer from those that are elicited by unwanted visual stimulation. First, we recorded control traces with UV illumination before the injection of caged $\mathrm{Ca}^{2+}$ buffer. Alternatively, the flash lamp system was used with long-wavelength filtering $(\lambda>475 \mathrm{~nm})$. This filtering produced equally strong visual excitation but no photorelease of caged $\mathrm{Ca}^{2+}$ buffer.

Relative cytosolic $\mathrm{Ca}^{2+}$ concentration changes in single TCs were monitored by epifluorescence imaging of Oregon-green 488 BAPTA1 emission. We used long working-distance objectives (Achroplan 20x NA $0.50 \mathrm{~W}$ and $40 \times$ NA $0.75 \mathrm{~W}$, Zeiss, Oberkochen, Germany) and an upright fixed-stage microscope (filter settings: excitation $475 \pm 20 \mathrm{~nm}$, dichroic mirror $500 \mathrm{~nm}$, emission $530 \pm 20 \mathrm{~nm}$; Axioskop FS, Zeiss) equipped with a cooled frame-transfer chargecoupled device camera (Quantix 57; Photometrics, Tucson, AZ, USA), operated with $4 \times 4$ pixel binning at a frame rate of $3 \mathrm{~Hz}$.

\section{Visual stimulation}

Light-emitting diode (LED) boards were used to present moving highcontrast square wave gratings in the receptive field of TCs. In the experiments performed in bridged recording mode, green LEDs were used and the angular extent of the pattern was approximately $50^{\circ} \times 60^{\circ}$, with the larger extent perpendicular to the axis of motion (for details see Kurtz et al., 2001). The temporal frequency of pattern motion was $4 \mathrm{~Hz}$. In the experiments with voltage clamp the LED matrix consisted of $48 \times 48$ ultra bright blue LEDs with a diameter of $3 \mathrm{~mm}$. The LED board was obliquely aligned at a $45^{\circ}$ angle to the $0^{\circ}$ azimuth of the fly and covered a visual field from about $-10^{\circ}$ to $70^{\circ}$ horizontally and from about $-45^{\circ}$ to $25^{\circ}$ vertically. The stimuli consisted of square-wave gratings with a spatial wavelength of about $25^{\circ}$ moving in the preferred direction of the neuron with a temporal frequency of about 3.125 cycles/s. The mean luminance as seen by the fly changed according to view angle and ranged from $400 \mathrm{~cd} / \mathrm{m}^{2}$ $\left(45^{\circ}\right.$ angle) to $5000 \mathrm{~cd} / \mathrm{m}^{2}\left(0^{\circ}\right.$ angle).

\section{Data analysis}

All electrophysiological data acquisition and stimulation and the control of visual stimulation were performed using self-written 
software (C, Borland, Scotts Valley, CA, USA and Visual C++ 6.0, Microsoft, Redmond, WA, USA) on a standard personal computer. All electrophysiological data analysis was performed off-line using custom analysis routines written in MatLab (The MathWorks, Natick, MA, USA).

The $\mathrm{Ca}^{2+}$ concentration signals were evaluated as fluorescence changes of the $\mathrm{Ca}^{2+}$-sensitive dye relative to resting values obtained from the first image $(\Delta \mathrm{F} / \mathrm{F})$. Background values were calculated by averaging the signal from unstained regions in the images and subtracted from the values in the regions of interest. This procedure adds noise to the $\Delta \mathrm{F} / \mathrm{F}$ time-courses but improves the comparability of $\mathrm{Ca}^{2+}$ signals from regions with different staining intensity. PMIS (GKR Computer Consulting, Boulder, CA, USA) was used for chargecoupled device camera control and image analysis. Matlab was used for further image data analysis.

Unless otherwise mentioned, all values are given as mean \pm SD. The lowercase letter ' $n$ ' denotes the number of measurements from one cell and uppercase ' $N$ ' denotes the number of cells.

\section{Results}

The HS neurons respond to motion in their preferred direction, i.e. front-to-back in the ipsilateral visual field, with graded and spike-like depolarizations ('spikelets') of their axonal membrane. Following cessation of motion, the membrane potential falls to a value several millivolts below the resting value and requires several seconds to recover (Fig. 1A). This AHP is employed here as an indicator of physiological mechanisms that are activated during motion stimulation in the preferred direction preceding the AHP and lead to directionselective adaptation. To characterize these mechanisms underlying the AHP, we tested whether membrane depolarization alone in the absence of synaptic input is sufficient to elicit an AHP. This was analysed by injecting depolarizing current pulses into HS neurons. In line with previous results, a $4 \mathrm{~s}$ motion stimulus in the preferred direction was effective at producing an AHP in all tested HS and $\mathrm{CH}$ cells (see example cell shown in Fig. 1A). When, in the same sample of cells, a membrane depolarization was generated in the absence of visual stimulation by a $4 \mathrm{~s}$ pulse of depolarizing current $(5 \mathrm{nA})$, an AHP of similar magnitude and with a similarly long recovery time-course in the range of seconds was elicited (see Fig. 1B for an example).

\section{Quantitative comparison of after-hyperpolarizations following visual stimulation and current injection}

The AHPs elicited by current injection and by visual stimulation differed in two respects. A much larger apparent depolarization was
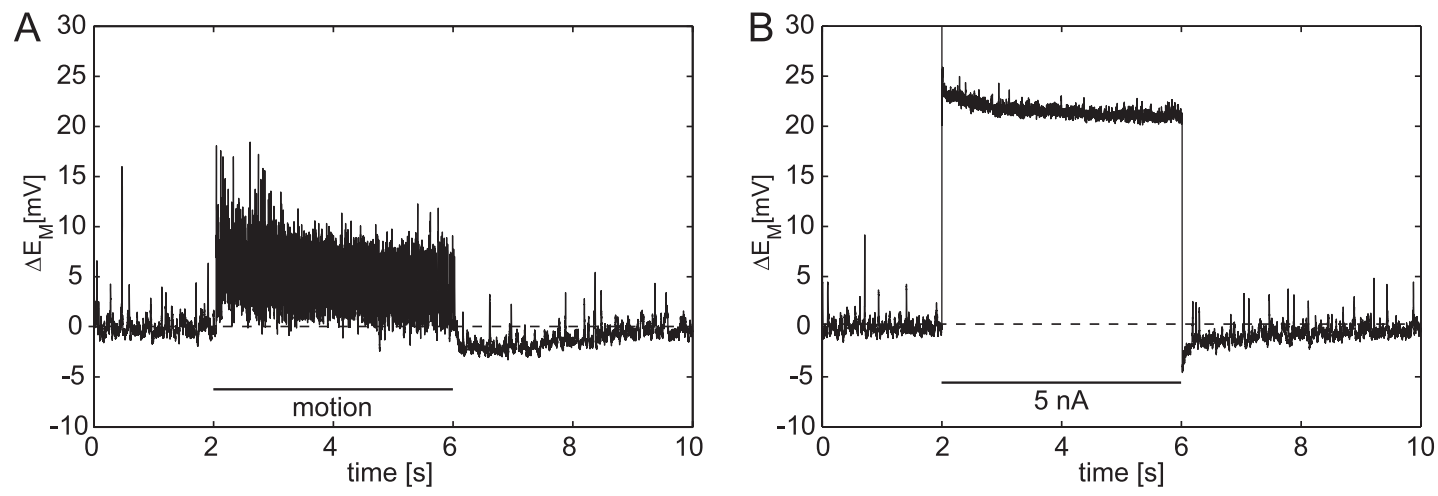

C

D
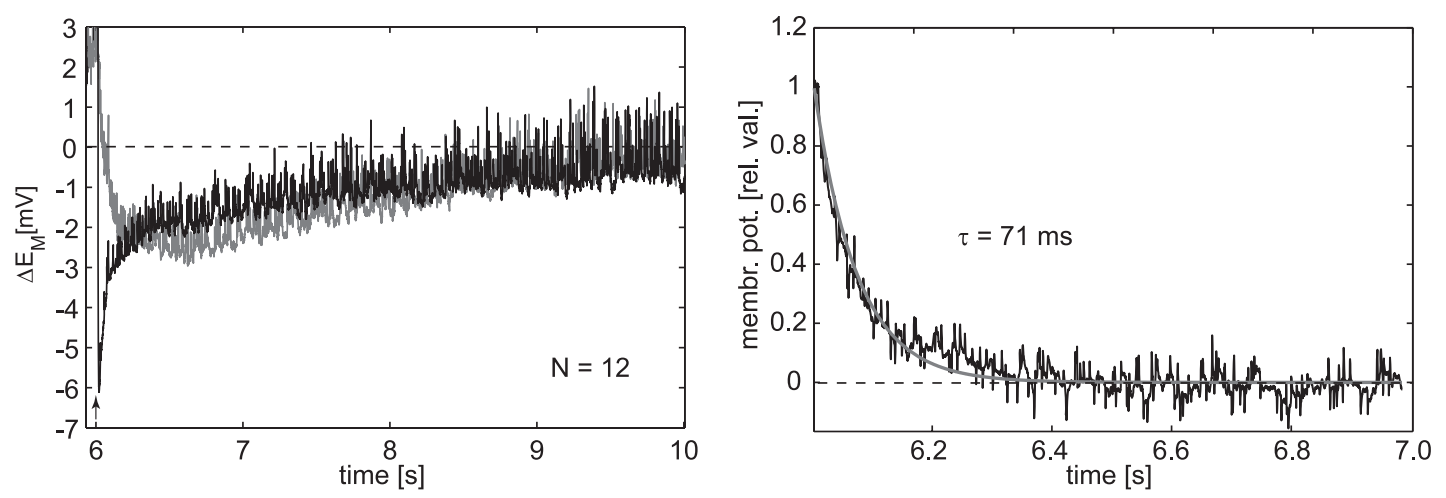

FIG. 1. AHPs following visual stimulation and current injection. (A) Response recorded from the axon of an HS cell to motion of a square-wave grating in the preferred direction, front-to-back in the ipsilateral visual field. The axonal response of the neuron during pattern motion consists of a graded depolarization with superimposed 'spikelets'. After the cessation of motion the membrane potential shifts to a hyperpolarized value and recovers to the resting potential after about 2-3 s. Dashed line, resting potential calculated as mean value in a $1 \mathrm{~s}$ time window preceding stimulation. (B) A prominent AHP is also present after depolarization of the same neuron by $5 \mathrm{nA}$ axonal current injection. (C) The time-courses of AHPs following visual stimulation (gray) and current injection (black) are plotted on a finer temporal scale. Mean traces obtained from the same sample of HS neurons $(N=12)$ are shown for the two conditions. Initially after stimulation (stimulus offset indicated by arrow), there is a faster transition to a hyperpolarized value after current injection than after visual stimulation. During later phases the time-courses match more closely. (D) Time-course of the difference between the membrane potentials following visual stimulation and current injection. The voltage trace in the first second after current injection was subtracted from that after visual stimulation. Mean values in the time windows 18-23 ms and 0.9-1 s after stimulus offset were used for normalization to one and to zero, respectively. A single-exponential decay function was fitted to the curve by a least-squares algorithm. The fit (gray line) yielded a decay time constant of $71 \mathrm{~ms}$, which fits well to estimations made for the low-pass filter of peripheral motion detectors in the adapted state (see Results). 
required to elicit an AHP by current injection than by visual stimulation. A plausible reason for this discrepancy is the large electrotonic distance of the recording site from the dendritic input region (see Discussion). In the example cell the measured depolarization during $5 \mathrm{nA}$ current injection was $20 \mathrm{mV}$ (Fig. 1B). Injection of current pulses weaker than $5 \mathrm{nA}$ did not consistently generate an AHP in all neurons (data not shown). In contrast, the same example neuron as shown in Fig. 1A depolarized in response to preferreddirection motion to only about $5 \mathrm{mV}$, except for the 'spikelets', which reached an amplitude of about $15 \mathrm{mV}$ (Fig. 1A). On average, during visual stimulation $\mathrm{HS}$ cells depolarized by $5.7 \pm 3.4 \mathrm{mV}$ (mean $\pm \mathrm{SD}$, $N=12$, averaged in a $3.8 \mathrm{~s}$ time window starting $0.2 \mathrm{~s}$ after motion onset) from resting level (determined in a $1 \mathrm{~s}$ time window before stimulation) and $\mathrm{CH}$ cells depolarized by $4.7 \pm 2.6 \mathrm{mV}(N=7)$. In the same sample of neurons, $5 \mathrm{nA}$ current injection elicited a depolarization of $40.7 \pm 21.8 \mathrm{mV}(N=12)$ and $69 \pm 31.0 \mathrm{mV}(N=7)$ from resting level in $\mathrm{HS}$ and $\mathrm{CH}$ cells, respectively.

The second difference between AHPs elicited by visual stimulation and those elicited by current injections concerns their time-courses. Although the slow recovery from the hyperpolarized state to resting membrane potential looks similar after visual stimulation and after current injection (Fig. 1C), the initial transition from the depolarized level during stimulation to the hyperpolarized level after stimulus offset is much faster after current injection than after visual stimulation. To trace possible reasons for this discrepancy, we subtracted the mean response after visual stimulation from that after current injection and analysed the time-course of the resulting difference trace (Fig. 1D). The difference was largest directly after stimulus offset and showed a decline that was fairly well fit by an exponential decay function. We suggest a simple explanation for this finding: with current injection the transition from the depolarized state to the slightly hyperpolarized state immediately after stimulus offset is shaped by the neuronal time constant alone. In contrast, with additional visual stimulation, much longer time constants have to be considered, affecting the decay time-course of the membrane potential after stimulus offset. These time constants can be attributed to motion detectors, which are thought to serve as input elements of TCs (Borst \& Egelhaaf, 1989; Egelhaaf \& Borst, 1989). When fitting an exponential decay function to the difference trace we obtained a time constant of $71 \mathrm{~ms}$ (Fig. 1D). This value is well within the range of values that have been derived from experimental studies that aimed to characterize the time constant of peripheral motion detectors in the adapted state (de Ruyter van Steveninck et al., 1986; Borst \& Egelhaaf, 1987; Harris et al., 1999).

In summary, the discrepancy in time-course between after-responses following visual stimulation and those following current injection can be well explained by the properties of peripheral processing structures, which are activated during visual stimulation but not during current injection.

We quantified the strength of the AHP following motion in the preferred direction and current injection by averaging the membrane potential in a $2 \mathrm{~s}$ time window starting at $200 \mathrm{~ms}$ after stimulus offset, i.e. when the initial difference in time-course between the two conditions is largely settled (cf. Fig. 1D). In all recorded HS and $\mathrm{CH}$ cells an AHP was present after visual stimulation as well as after current injection (Fig. 2). The amplitude of the AHP was similar in the two cell types. The above results demonstrate that in fly TCs an AHP similar to that following stimulation with preferred-direction motion is also reliably evoked when a depolarization is artificially generated by current injection.

\section{Depolarizing voltage-clamp steps elicit a slowly activated and slowly recovering outward current}

As a further approach to test whether AHPs evoked by visual stimulation and by artificial depolarization, respectively, are likely to result from the same cellular mechanisms we applied a depolarizing voltage-clamp rectangular step protocol. The voltage of VS neurons was stepped from a resting value, as obtained from the recording resting potential in bridged mode, to a $3.8 \mathrm{~s}$ rectangular depolarization of $25 \mathrm{mV}$ and then back to the resting value (Fig. 3A). To extract the currents underlying the AHP we analysed the current that is injected into the cell to maintain the depolarized holding potential and the current after switching back from the depolarizing voltage step. In most neurons the outward current during the depolarizing step command increased slowly. In six out of seven neurons the amplitude of the current was larger at the end of the voltage step than at its beginning (Fig. 3B). This finding indicates that an input resistance change resulting in a slowly activating outward current is elicited by long-lasting depolarization. Following the step from the depolarized level back to the resting value the clamp current initially attained a value more positive than that before the depolarization. This outward current recovered slowly within several seconds. It is plausible to assume that this after-current corresponds to the current that builds up visual stimulation

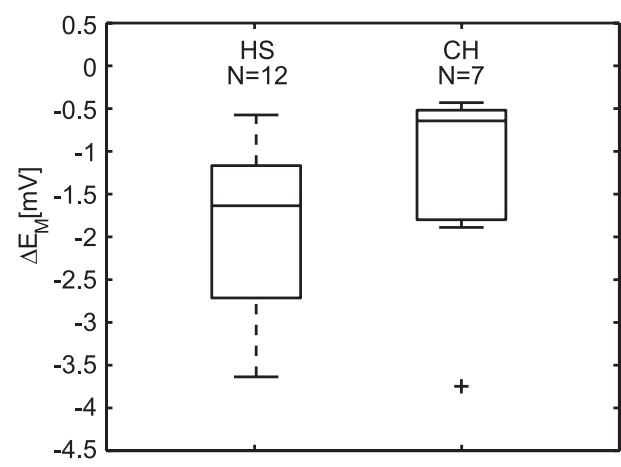

current injection

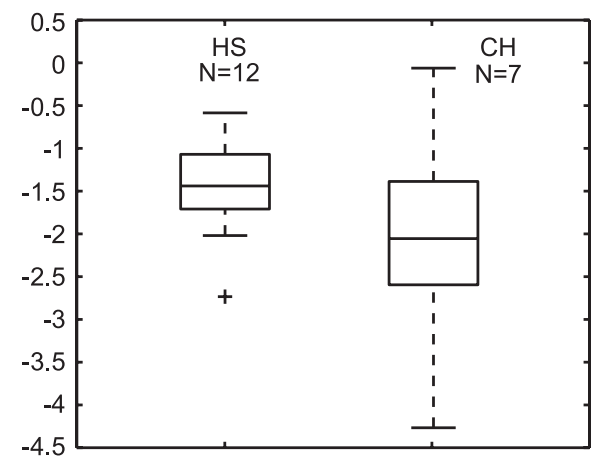

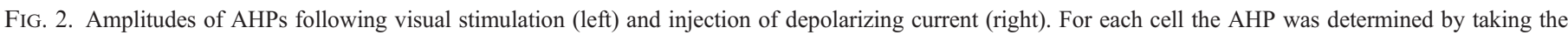

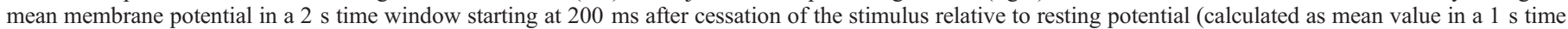

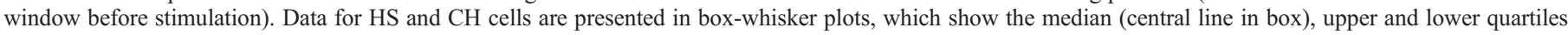

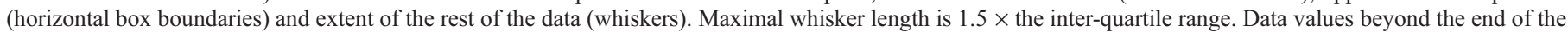
whiskers are classified as outliers and are displayed as crosses. 

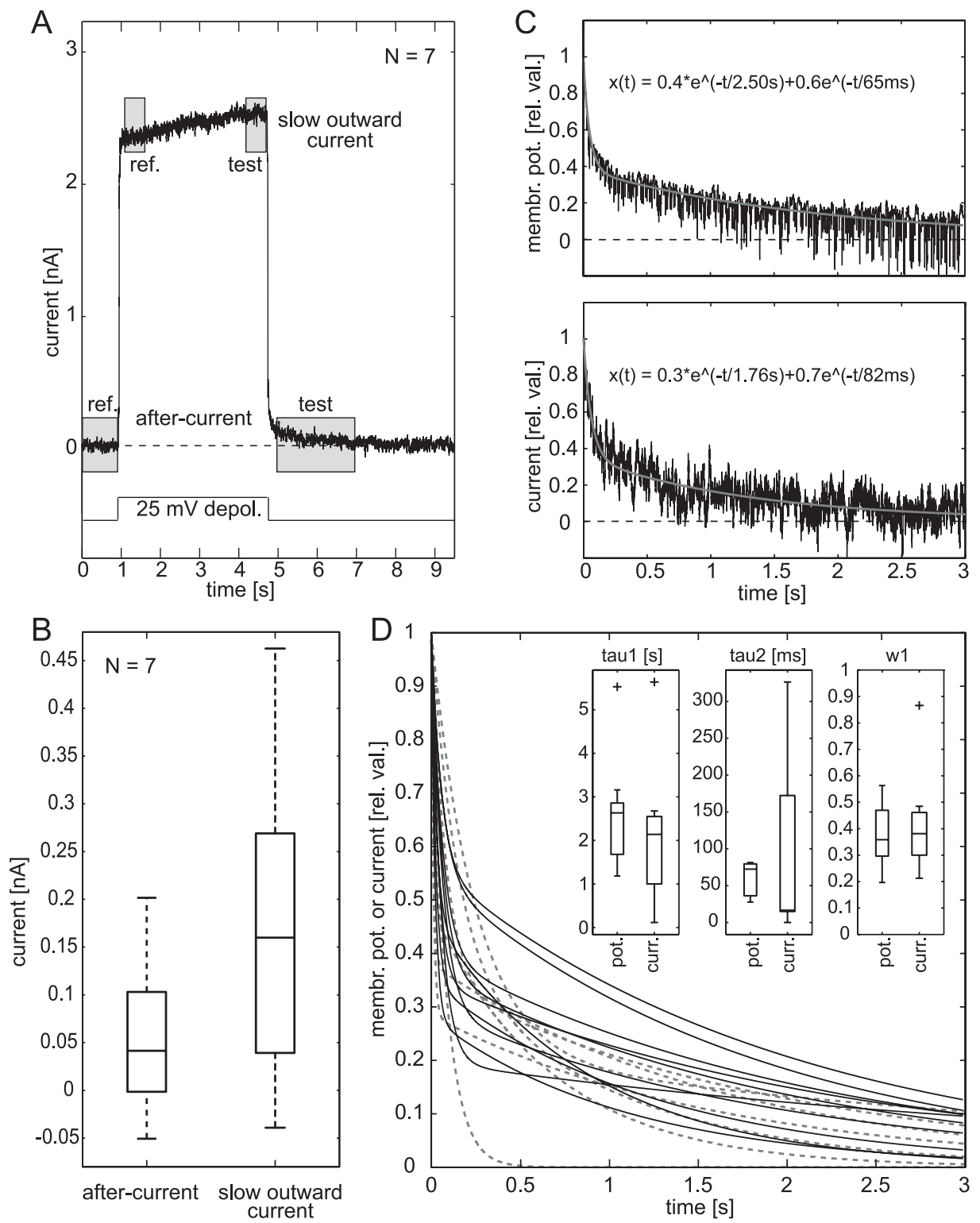

FIG. 3. Sustained depolarization elicits a slow outward current. (A) Mean current trace of VS neurons $(N=7)$ during a depolarizing voltage-clamp protocol. A $3.8 \mathrm{~s}$ rectangular depolarization by $25 \mathrm{mV}$ relative to resting potential was applied. (B) The difference between the mean current in a $0.5 \mathrm{~s}$ time window at the end of the voltage command and that in a corresponding time window at the beginning of the command is calculated (left boxplot, 'slow outward current'; see shaded areas in A for illustration of time windows). The current following the depolarization (right boxplot, 'after-current') is quantified equivalent to the AHP by taking the average in a $2 \mathrm{~s}$ time window starting at $200 \mathrm{~ms}$ after the depolarization and subtracting mean resting current $(0.9 \mathrm{~s}$ time window before depolarization). See Fig. 2 for details on box-whisker plots. (C) Comparison of the mean time-course of the AHP following injection of depolarizing current with the mean time-course of the after-current following a depolarizing voltage-clamp command. The data traces are the same as those shown in Figs $1 \mathrm{~B}$ and $\mathrm{C}$ and $3 \mathrm{~A}$, respectively. Note that the voltage trace appears inverted for easier comparison with the current trace. Mean values in the time windows 18-23 and 5-10 ms after stimulus offset were used for normalization to one of the voltage trace and the current trace, respectively. A later time window for normalization of the voltage trace is required to account for the fact that the voltage has to make a large step from the depolarized level during current injection to the subsequent AHP (see Fig. 1B). (D) Fitting results for individual cells. Solid black lines represent fits to the AHP, dotted gray lines represent fits to after-currents. Same normalization as used with mean traces. The inset shows box-whisker plots of the two exponential time constants (tau1 and tau2) and the weight of the first time constant (w1) for AHP ('pot.', $N=10$ ) and current ('curr.', $N=7$ ). Two of the AHP time-courses were excluded from this analysis because average values in the normalization time window were close to zero, resulting in large fluctuations of the normalized time-course and inability to fit with a decaying double-exponential function.

slowly during the depolarization. As done before for the AHP following visual stimulation and current injection, we quantified the after-current in a time window of $2 \mathrm{~s}$ beginning at $200 \mathrm{~ms}$ after the depolarization. In six out of seven neurons the current in this time window had a positive value, consistent with a current that would be required to cause an AHP.

To investigate whether the outward current after depolarizing voltage-clamp commands and the AHP following depolarizing current injection are likely to result from a common ionic conductance we analysed their time-courses in more detail. The mean AHP after depolarizing current injection was fitted by the weighted sum of two exponential functions (Fig. 3C, top). The fast component with a time constant of $65 \mathrm{~ms}$ might correspond to a voltage-activated potassium outward current (Haag et al., 1997) or to another current causing fast AHP. Although this component would settle too rapidly to account for long-lasting AHP, the second, slow exponential function has a time 
constant of $2.50 \mathrm{~s}$, which corresponds well to a slowly recovering outward current. The mean outward current after depolarizing voltageclamp commands followed a similar time-course (Fig. 3C, bottom). The exponential fit yielded slow and fast time constants of the exponential functions of $82 \mathrm{~ms}$ and $1.76 \mathrm{~s}$, respectively. We fitted the voltage and current traces of individual cells by double-exponential functions to analyse whether their time-courses showed systematic differences (Fig. 3D). The temporal profiles of the fits showed a large overall variability but did not differ systematically between fits to AHPs (black lines) and fits to after-currents (dotted gray lines). Accordingly, the distributions of exponential time constants obtained from the fits to AHPs and after-currents cover a broad range of values and produce large overlaps between the two conditions (Fig. 3D, inset). The similarity of the time-courses of AHPs and after-currents suggests that the same activity-dependent conductance is activated by depolarizing current injection and by voltage clamp to a depolarized level.

\section{Voltage clamp to resting potential during visual stimulation leaves after-hyperpolarization unaffected}

The results presented in the previous sections strongly suggest that AHP in fly TCs is based on intrinsic depolarization-activated mechanisms. We therefore hypothesized that the AHP or the corresponding outward current might be attenuated when neuronal depolarization during visual stimulation is artificially blocked. In one approach we first recorded neurons in bridged mode to determine their resting potential. With the start of preferred-direction motion the recording mode was switched to voltage clamp and the axonal membrane potential was held at the neuron's resting potential (Fig. 4A, black trace). When pattern motion stopped we switched back to bridged recording to monitor the AHP and to compare its magnitude with that obtained in control trials (gray trace) in which the bridged mode was used throughout the entire recording. Contrary to our expectations the AHP was similarly strong under both conditions.

In a second approach we recorded currents when neurons were voltage clamped to their resting potential throughout the recording, i.e. before, during and after presentation of preferred-direction motion (Fig. 4B, black trace). In the control condition neurons were voltage clamped only before and after visual stimulation but were recorded in bridged mode during visual stimulation (gray trace). We addressed two aspects: first, whether the typical, slowly recovering outward current observed after depolarizing voltage-clamp commands is also present after preferred-direction motion and second, if present, this current can be compared between the test and the control condition to find out whether it is attenuated when the axonal depolarization during visual stimulation is blocked. We found that an outward current that slowly recovers after visual stimulation is present after stimulation with preferred-direction motion. The time-course of this current differed clearly from that after rectangular depolarizing voltage-clamp commands during the first tens of milliseconds after stimulation but not in later phases (cf. Figs $3 \mathrm{~A}$ and $\mathrm{C}$, and 4B). This difference is consistent with a similar discrepancy in time-courses of AHPs following visual stimulation on the one hand and depolarizing currents on the other (see Fig. 1C). In analogy, the different timecourses of the currents can plausibly be attributed to the temporal properties of correlation-type motion detectors, which are activated during visual stimulation but not during depolarizing voltage clamp. Thus, the slowly recovering outward currents after visual stimulation are probably the same as those after depolarizing voltage clamp. Similar to what we found for the AHP, this current was not attenuated when the visual response was blocked by voltage clamping the cells to their resting potentials.
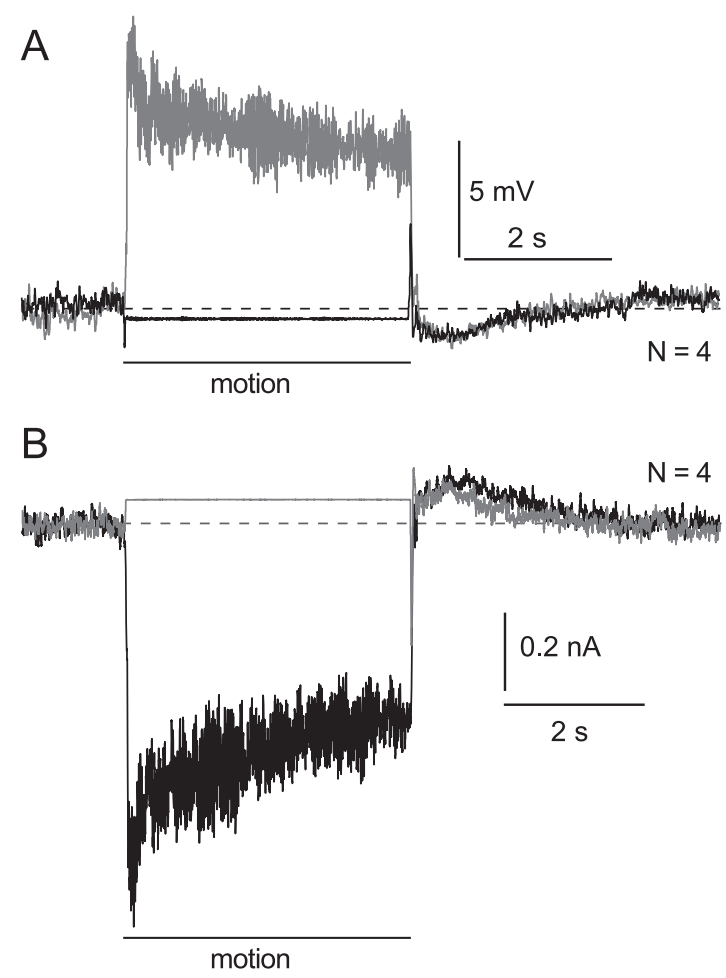

FIG. 4. Experimental tests of whether elimination of axonal membrane potential responses to visual stimulation affects after-responses. (A) Mean responses of VS neurons $(N=4)$ to motion in the preferred direction when recorded in bridge mode (gray) and when recorded in bridge mode before and after pattern motion but with voltage clamp to resting potential during pattern motion (black). Dashed line, resting potential. (B) Mean currents of VS neurons (same cells as in A) during preferred-direction motion when the cells were voltage clamped to their resting potential (black) and when the voltage clamp was switched to bridge mode during pattern motion (gray). Dashed line, current during resting potential.

\section{Testing for a potential role of $\mathrm{Ca}^{2+}$ in the generation of an after-hyperpolarization}

The membrane currents underlying the AHP may be regulated by $\mathrm{Ca}^{2+}$ entering the cytosol of TCs via voltage-dependent $\mathrm{Ca}^{2+}$ channels (Borst \& Egelhaaf, 1992; Haag \& Borst, 2000). In general, $\mathrm{Ca}^{2+}$ presents a plausible regulator of activity-dependent adaptation because the dynamics of cytosolic $\mathrm{Ca}^{2+}$ concentration changes during visual stimulation have a similarly slow time-course as the build-up of AHP by sustained stimulation and as its recovery after cessation of stimulation (Kurtz et al., 2000). However, a role of $\mathrm{Ca}^{2+}$ in motion adaptation of TCs was rendered unlikely because in a previous study artificial elevation of cytosolic $\mathrm{Ca}^{2+}$ did not elicit adaptation effects (Kurtz, 2007). To re-examine whether the AHP is $\mathrm{Ca}^{2+}$ dependent in fly TCs, in the present study we used an approach that is complementary to artificial $\mathrm{Ca}^{2+}$ release, namely artificial buffering of cytosolic $\mathrm{Ca}^{2+}$ (see Discussion). $\mathrm{A} \mathrm{Ca}^{2+}$ buffer (Diazo-2) that can be rapidly activated by a flash of UV light was used because this approach avoids long delays between traces recorded under different buffer conditions. Such delays, which are unavoidable when using iontophoretic filling of cells with conventional $\mathrm{Ca}^{2+}$ buffers like BAPTA, would make it difficult to distinguish the effects of the $\mathrm{Ca}^{2+}$ buffer from gradual shifts of neuronal properties over time or changes in recording quality. Figure $5 \mathrm{~A}$ shows the voltage response to preferred-direction motion of an HS cell filled with the $\mathrm{Ca}^{2+}$ buffer but 
A

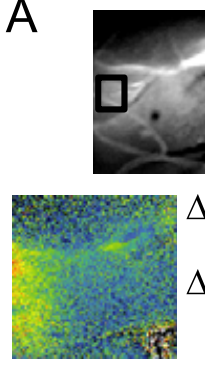

B

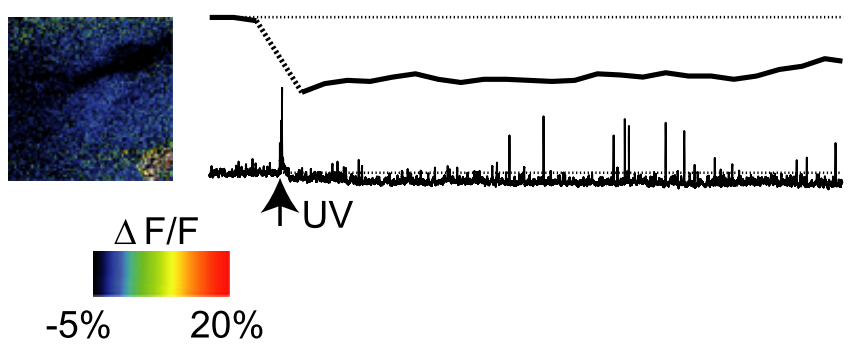

C
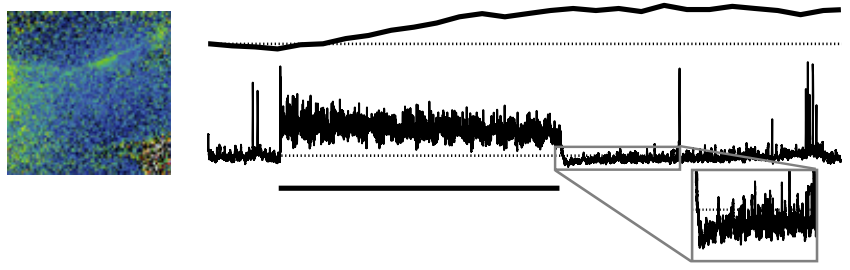

D

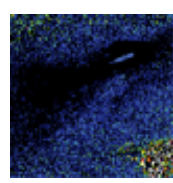

E
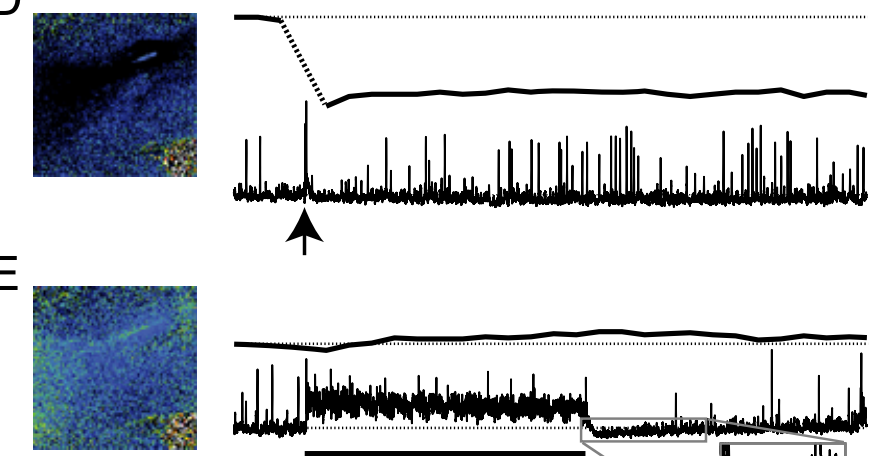
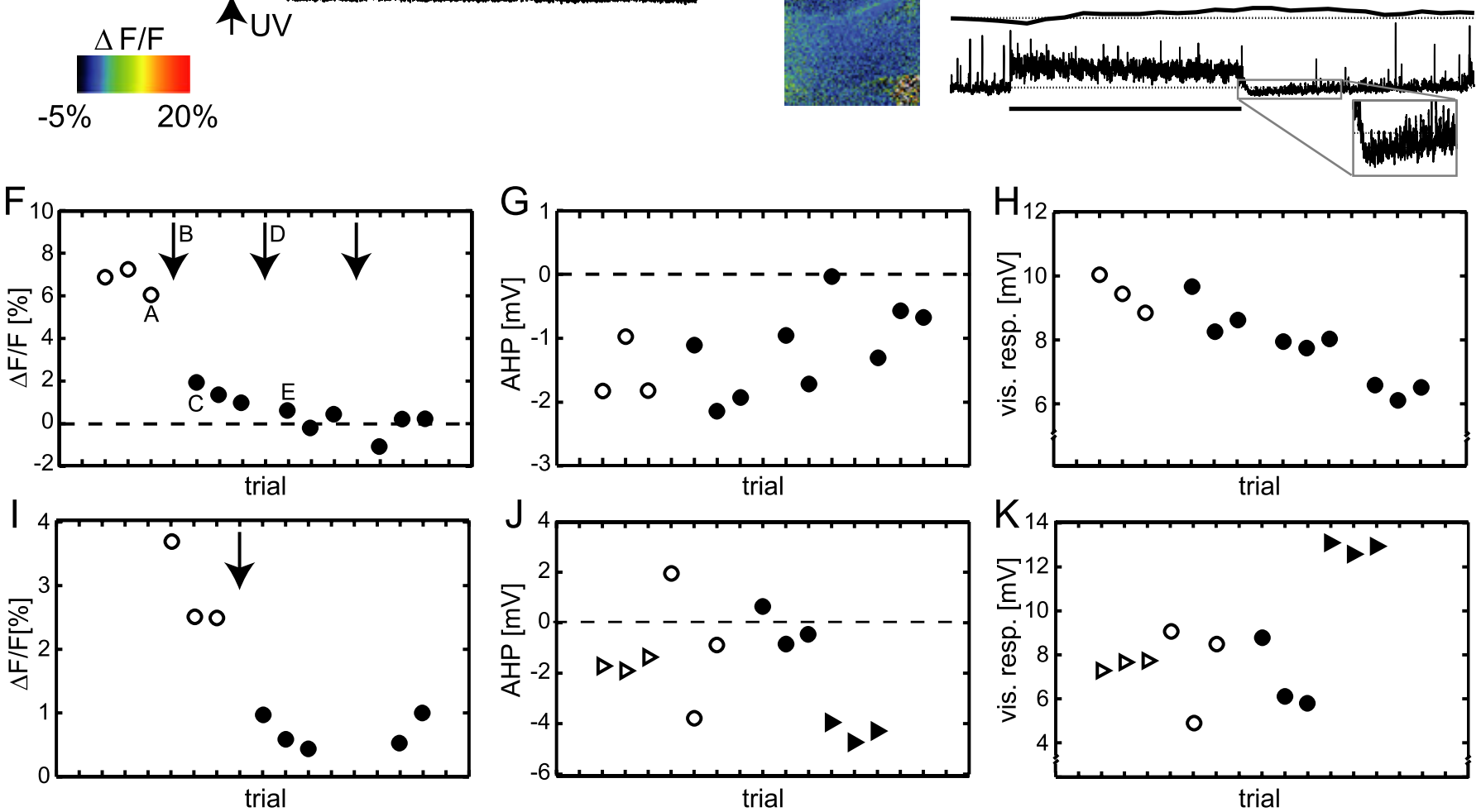

FIG. 5. Insensitivity of AHP to artificially increased buffering of cytosolic calcium. (A) Change in membrane potential $\left(\Delta \mathrm{E}_{\mathrm{M}}\right)$ and cytosolic calcium signal $(\Delta \mathrm{F} / \mathrm{F})$ of an HS neuron during visual motion in the preferred direction. The neuron was filled with the fluorescent calcium indicator Oregon-green BAPTA-1 and the caged calcium buffer Diazo-2. The black box in the raw fluorescence image (top left) indicates the area in which fluorescence signals shown in F were averaged. The white box shows the region used for background subtraction of the fluorescence values. The color-coded image depicts the background-corrected relative fluorescence $(\Delta \mathrm{F} / \mathrm{F})$ averaged over the stimulation period. In the inset of the membrane potential trace part of the response is shown with the amplitude axis enlarged by a factor of 4 to illustrate the presence of an AHP. (B) Activation of Diazo-2 by a UV flash (arrow) causes a decrease in $\Delta \mathrm{F} / \mathrm{F}$, indicative of a rapid decline in the cytosolic calcium concentration. Color-coded image calculated for the same time window as in A. (C) Subsequent visual stimulation leads to a weak calcium signal but still to a prominent AHP. (D and E) After a further cycle of Diazo-2 activation motion-evoked calcium signals are nearly abolished but the AHP is still present. (F) Background-corrected relative fluorescence $(\Delta \mathrm{F} / \mathrm{F})$ averaged over stimulation periods during successive trials. The arrows indicate the time-points of Diazo-2 activation. Open and closed circles denote data obtained before and after activation of Diazo-2, respectively. Lettering refers to the corresponding data traces shown in A-E. (G) AHPs quantified in $2 \mathrm{~s}$ time windows starting at $200 \mathrm{~ms}$ after cessation of motion. (H) Visual responses averaged over the period of visual motion stimulation. (I-K) Data obtained from a second HS neuron, presented in the same way as before. Some of the electrical recordings were performed without simultaneous imaging because fluorescence light for excitation of Oregon-green BAPTA-1 causes tonic photoreceptor activation and a corresponding attenuation of responses to the visual motion stimulus. Circles and triangles denote data obtained with and without fluorescence excitation, respectively.

before its activation by UV light. The visual response was followed by an AHP. As the neuron was also filled with a $\mathrm{Ca}^{2+}$-sensitive dye, an increase in the cytosolic $\mathrm{Ca}^{2+}$ concentration during motion led to an increase in the background-corrected relative fluorescence signal $(\Delta \mathrm{F} / \mathrm{F})$ in the region of the dendrite. Activation of the $\mathrm{Ca}^{2+}$ buffer by a
UV flash caused a quick downward step in the fluorescence signal, indicative of a decline in the resting cytosolic $\mathrm{Ca}^{2+}$ concentration (see Fig. 5B). Moreover, the increased cytosolic $\mathrm{Ca}^{2+}$ buffer capacity led to a strongly reduced $\mathrm{Ca}^{2+}$ signal when preferred-direction motion is presented in the subsequent trial (Fig. 5C). Nevertheless, the voltage 
response was still followed by a prominent AHP. After a further increase in cytosolic $\mathrm{Ca}^{2+}$ buffer capacity by a second UV flash (see Fig. 5D), motion responses were still followed by an AHP, although the $\mathrm{Ca}^{2+}$ signal was almost entirely abolished (see Fig. 5E). We quantified the amplitude of the AHP by averaging the membrane potential in a $2 \mathrm{~s}$ time window starting at $200 \mathrm{~ms}$ after motion offset and compared it with the amplitude of the $\mathrm{Ca}^{2+}$ signal, averaged over the duration of the visual stimulus (Fig. 5F and G). The first UV flash caused a strong reduction of the $\mathrm{Ca}^{2+}$ signal to less than one-third but no change in the strength of the AHP. After the second flash, the $\mathrm{Ca}^{2+}$ signal was near zero. AHPs were still present but there was a gradual decline in their amplitude towards the end of the recording. However, a similar decrease was also present in the voltage response during motion stimulation, suggesting that they either resulted from a shift in neuronal sensitivity or from a decline in recording quality (see Fig. 5H). Thus, the decline in AHP amplitude is probably not a specific effect of the artificially increased $\mathrm{Ca}^{2+}$ buffer capacity. One further argument speaks against a $\mathrm{Ca}^{2+}$-dependent regulation of the AHP. If the underlying currents were tonically activated by resting $\mathrm{Ca}^{2+}$, the photoactivation of a $\mathrm{Ca}^{2+}$ buffer should cause a shift to a slightly depolarized membrane potential. Instead, the UV flashes caused, if anything, a weak, long-lasting hyperpolarization (see Fig. 5B). Note that the brief depolarization initially after the UV flash results from the sensitivity of fly photoreceptors to UV light (see Fig. 5B and D; cf. Kurtz, 2004, 2007). The absence of an effect of UV-activated $\mathrm{Ca}^{2+}$ buffer on the AHP was corroborated in one more HS neuron (see Fig. 5I-K).

\section{Discussion}

In fly TCs an AHP is generated after cessation of motion in the preferred direction (Harris et al., 2000; Kurtz et al., 2000). In the present study we have shown that this AHP is regulated by membrane depolarization. This is indicated by two findings. First, artificial depolarization by current injection in the absence of visual motion was sufficient to generate an AHP. Second, a slowly activating outward current developed during sustained depolarizing command potentials. Similar to the slow recovery from AHP, this current recovered on a time-scale of seconds after stepping back to resting potential. It will be discussed that the mechanisms underlying AHP manifest themselves during visual motion stimulation preceding the AHP and cause direction-selective motion adaptation.

\section{Putative mechanisms underlying after-hyperpolarization in tangential cells}

In general, several different mechanisms, working in isolation or in combination, may account for the generation of an activity-dependent AHP. The results reported in the present and in previous studies help to evaluate the relevance of these putative mechanisms in fly TCs.

The putative mechanisms underlying AHP can be segregated into processes that are located presynaptically to TCs and those that are cell intrinsic. With respect to presynaptic mechanisms it has to be considered that fly TCs receive input from acetylcholinergic synapses, which cause depolarization of the TC, and from GABAergic synapses, which lead to hyperpolarization (see Fig. 6). Motion in the preferred direction leads to a strong activation of excitatory (acetylcholinergic) inputs and might cause an attenuation of these inputs after sustained stimulation. Such presynaptic adaptation, or simple fatigue, will result in an AHP of the TC if the activity of excitatory inputs remains below resting activity for some time after the cessation of preferred-direction motion (see e.g. Manookin \& Demb, 2006). However, an entirely presynaptic origin of the AHP in TCs is not compatible with the finding of the present study that the AHP can be generated by depolarization of the TC in the absence of visual stimulation. Moreover, it was shown in a previous study that AHP is associated with an increase in membrane conductance (Kurtz, 2007). This finding implied that the cellular mechanism underlying AHP is an activityregulated ionic current. Attenuation of synaptic inputs during sustained motion stimulation would have the opposite effect, namely a decrease in conductance of the TC. The same holds true for a desensitization of acetylcholine receptors in the postsynaptic membrane. Thus, the present study and Kurtz (2007) indicate that an intrinsic biophysical mechanism leads to the AHP of TCs. This conclusion agrees with recent results obtained in HS neurons of the hoverfly Eristalis tenax. The AHP, when elicited by local stimulation, also affected the response to subsequent test stimuli when these were presented in a different part of the spatial receptive field (Nordström \& O’Carroll, 2009).

The putative cell-intrinsic mechanisms underlying AHP can be segregated into three classes. First, AHP might be regulated by a signaling pathway that is activated by the synaptic input but not directly dependent on membrane depolarization (see e.g. Knoflach \& Kemp, 1998; Dutar et al., 2000). Although such mechanisms might lead to a conductance increase and are thus compatible with Kurtz (2007), our present finding that membrane depolarization without activation of synaptic inputs causes AHP speaks against a major role of transmitter-activated regulatory mechanisms.

Second, the homeostatic maintenance of ionic gradients might lead to an AHP. Relevant in this context is, in particular, the activity of $\mathrm{Na}^{+} / \mathrm{K}^{+}$pumps, which has been shown to go along with long-lasting membrane hyperpolarization (see e.g. Jansen \& Nicholls, 1973; Jansonius, 1990; Gerster et al., 1997; Arganda et al., 2007). It is, however, improbable that the $\mathrm{Na}^{+} / \mathrm{K}^{+}$pump accounts for AHP in TCs because in the present study membrane depolarization alone without visual stimulation was shown to cause an AHP. In HS and VS neurons, which possess voltage-dependent $\mathrm{Na}^{+}$channels (Haag et al., 1997), depolarization alone can be expected to cause some $\mathrm{Na}^{+}$inflow but much less than visual stimulation, during which $\mathrm{Na}^{+}$enters the cell additionally via acetylcholine receptor channels. In $\mathrm{CH}$ cells an increase of the cytosolic $\mathrm{Na}^{+}$concentration during depolarization without visual stimulation is unlikely because this cell type lacks voltage-dependent $\mathrm{Na}^{+}$channels (Haag et al., 1997). In the present study, depolarizing current injection into $\mathrm{CH}$ was followed by an AHP that was similarly strong or even slightly stronger than in HS cells (see Fig. 2). This finding is incompatible with a prominent role of $\mathrm{Na}^{+} / \mathrm{K}^{+}$ pumps in the generation of an AHP.

Third, AHP might be regulated by membrane depolarization itself, either directly or via a mediator such as $\mathrm{Na}^{+}$or $\mathrm{Ca}^{2+}$ (see e.g. SanchezVives et al., 2000; Torkkeli et al., 2001; Peron \& Gabbiani, 2009). Although Haag et al. (1997) concluded that an $\mathrm{Na}^{+}$-dependent $\mathrm{K}^{+}$channel (for review see Bhattacharjee \& Kaczmarek, 2005) is present in HS cells, we think that a regulation of AHP via concentration changes in $\mathrm{Na}^{+}$is unlikely for the same reason as given above with respect to $\mathrm{Na}^{+} / \mathrm{K}^{+}$pumps, i.e. in $\mathrm{CH}$ cells injection of depolarizing current is followed by an AHP, although large rises in cytosolic $\mathrm{Na}^{+}$concentration are improbable because voltage-dependent $\mathrm{Na}^{+}$channels are missing in this cell type. A role of $\mathrm{Ca}^{2+}$ in the regulation of the AHP was proposed in a former study because the AHP is correlated with cytosolic $\mathrm{Ca}^{2+}$ accumulation in its magnitude and time-course (Kurtz et al., 2000). Contrary to this idea, in the present account we provide evidence that the AHP is unaffected by the cytosolic $\mathrm{Ca}^{2+}$ level. Visual stimulation was followed by an AHP even 


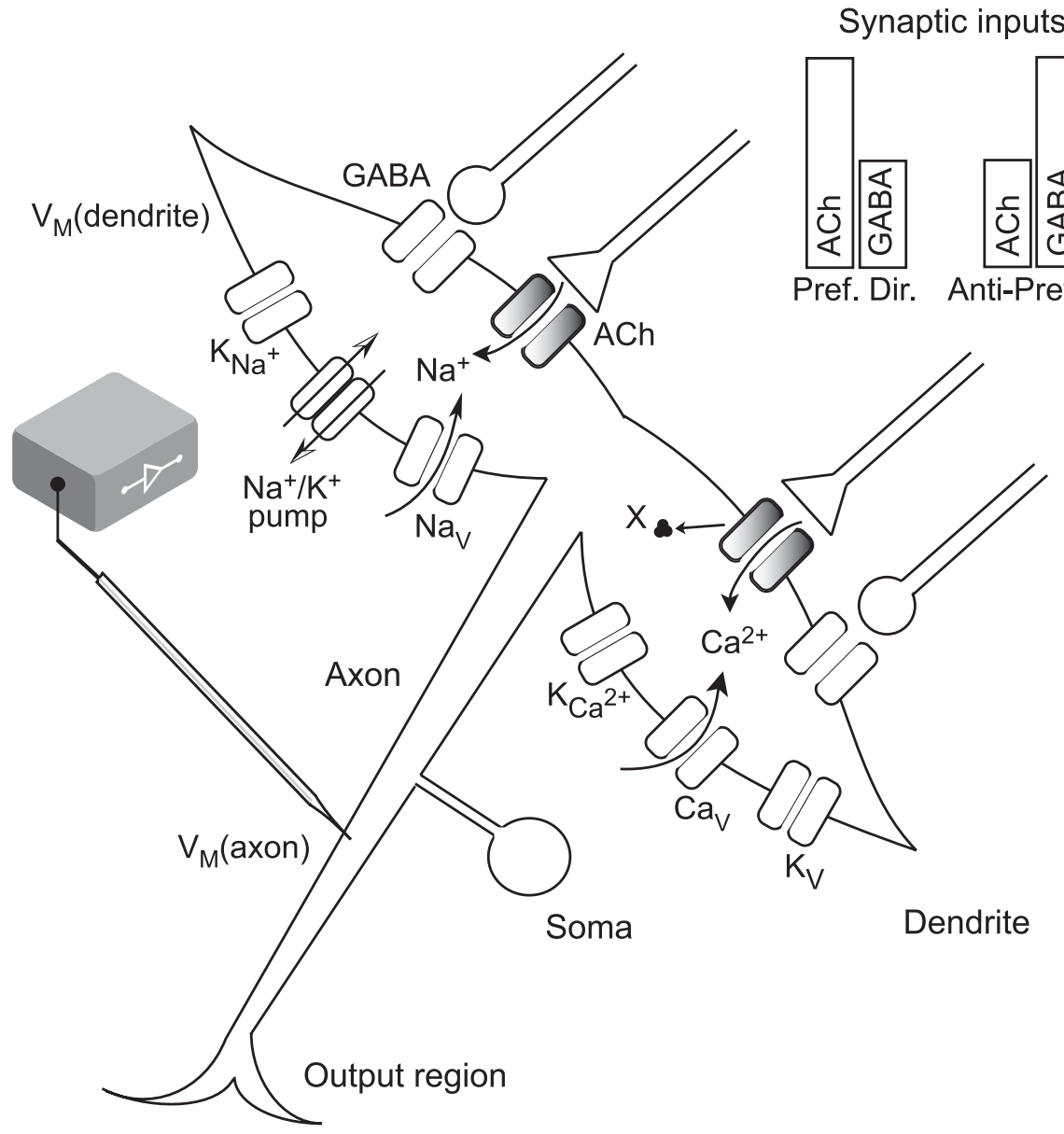

FIG. 6. Simplified scheme of a VS neuron illustrating hypothetical mechanisms that may contribute to the generation of an AHP. The neuron consists of a large, extensively ramified dendrite (arborization not detailed in scheme), an axon, an output region and a soma, which is connected to the axon via a thin neurite. Voltage recording and voltage clamp were performed during axonal penetrations. The output of local motion-sensitive elements converges on the dendrite in a retinotopic fashion, forming inhibitory GABAergic and excitatory acetylcholinergic synapses (two pairs of antagonistic input synapses shown in scheme). One putative cause for AHP after motion in the preferred direction is an activity-dependent reduction of acetylcholinergic inputs or synaptic desensitization. The other hypothetical mechanisms are intrinsic to the neuron. First, a signaling pathway is activated by acetylcholine receptors (termed X in the scheme) and leads to an AHP. Second, the $\mathrm{Na}^{+} / \mathrm{K}^{+}$pump might generate an AHP. Third, membrane depolarization might lead to AHP, either directly via slowly regulated ion channels (e.g. $\mathrm{K}^{+}$channels, shown in the model as $\left.\mathrm{K}_{\mathrm{V}}\right)$ or indirectly via $\mathrm{Ca}^{2+}$-dependent $\mathrm{K}^{+}$channels $\left(\mathrm{K}_{\mathrm{Ca}^{2+}}\right)$ or $\mathrm{Na}^{+}$-dependent $\mathrm{K}^{+}$channels $\left(\mathrm{K}_{\mathrm{Na}^{+}}\right)$. It is further indicated in the scheme that the cytosolic $\mathrm{Na}^{+}$concentration may be affected by both the synaptic activation of acetylcholine receptors and the opening of voltage-dependent $\mathrm{Na}^{+}$channels $\left(\mathrm{Na}_{\mathrm{V}}\right)$. Likewise, accumulation of cytosolic $\mathrm{Ca}^{2+}$ can be the result of $\mathrm{Ca}^{2+}$ inflow through acetylcholine receptor channels and through voltage-dependent $\mathrm{Ca}^{2+}$ channels $\left(\mathrm{Ca}_{\mathrm{V}}\right)$. Although only indicated in the dendrite, all ion channels and pumps apart from the transmitter-regulated channels might also be present in the axon. Upper right inset, sketch illustrating activation of acetylcholinergic and GABAergic synaptic inputs during motion in the preferred and anti-preferred direction (see Discussion for details). ACh, acetylcholine.

when $\mathrm{Ca}^{2+}$ accumulation was strongly reduced by a UV-activated $\mathrm{Ca}^{2+}$ buffer. This result is in accordance with our previous finding that an artificial increase of the cytosolic $\mathrm{Ca}^{2+}$ level by photolysis of caged $\mathrm{Ca}^{2+}$ did not produce an AHP or an increase in conductance, which typically accompanies the AHP (Kurtz, 2007). One caveat of our earlier $\mathrm{Ca}^{2+}$ photorelease experiments was, however, that $\mathrm{Ca}^{2+}$ might rise to much higher concentrations close to the cell membrane during visual stimulation than after photolysis of caged $\mathrm{Ca}^{2+}$. Thus, the $\mathrm{Ca}^{2+}$ concentration might not have reached a critical threshold for the induction of an AHP in the photolysis experiments of the former study. In contrast, $\mathrm{Ca}^{2+}$ buffering is increased in the approach of the present study. Threshold effects cannot explain why this manipulation should have no impact on the AHP because an AHP after visual stimulation clearly shows that a lower threshold has already been crossed. Thus, instead of threshold effects, a potential saturation of AHP magnitude with increased $\mathrm{Ca}^{2+}$ levels might render the photorelease of $\mathrm{Ca}^{2+}$ buffer ineffective. This is, however, unlikely in the range of visual stimuli used in the present study because larger AHP amplitudes can be reached by using longer stimulation periods (R. Kurtz, unpublished observations; see also Kurtz et al., 2000). Thus, even if $\mathrm{Ca}^{2+}$ buffering were less complete near the membrane than it appears to be when judged by inspection of fluorescence signals, at least some attenuation of the AHP would be expected if its magnitude were regulated by $\mathrm{Ca}^{2+}$.

In conclusion, together with the previous study (Kurtz, 2007), the present results leave the membrane depolarization itself as the most likely mechanism underlying AHP in TCs. Such a regulation could be mediated by an as yet unidentified messenger or by a voltagedependent conductance with very slow kinetic properties. In mammals, potassium channels with such properties, the KCNQ channels, form key regulators of neuronal excitability (Wang, 1998). A homolog of these channels has also been demonstrated to exist in Drosophila (Wen et al., 2005). Any further characterization of the physiological basis of AHP in TCs would almost certainly require pharmacological 
approaches. Unfortunately, we found drug application in our in-vivo preparation to exert only very slow and variable effects, which is probably a consequence of shielding of the neuropil by a dense neurolemma and of multiple, potentially antagonistic effects at different sites of the neuronal network.

For the regulation of AHP by membrane voltage, a depolarization of either the dendrite or the axon, or both, may be relevant, depending on the localization of the underlying ion channels. Compartmental modeling of fly TCs implied that local current injection leads to spatially non-uniform voltage responses, which is a consequence of their large, extensively arborized dendrites (Borst \& Haag, 1996). During simulated axonal current injection into a VS neuron model the voltage was estimated to drop by more than $70 \%$ in fine dendritic endings (Haag et al., 1997). In the present study we found that, during axonal current injection, much larger depolarization than during visual stimulation was necessary to generate an AHP of similar magnitude (cf. Fig. 1A and B). Considering the TC models, this result suggests that the depolarization-activated mechanism underlying AHP is localized in the dendrite. Moreover, the AHP as well as the corresponding outward current after preferred-direction motion were not noticeably affected when the neurons were voltage clamped to their resting potential during motion stimulation. At first sight, this appears to contradict our hypothesis that the AHP is based on an intrinsic, depolarization-dependent mechanism. However, as a result of poor space clamp, large dendritic depolarization to preferreddirection motion can be expected even when the axon is clamped to resting potential. Thus, rather than speaking against a regulation of AHP by depolarization in general, our results indicate that the underlying mechanism is not located in the axon but in the dendrite, probably close to the synaptic input sites. Consistent with this conclusion, the generation of 'spikelets' appears not to be necessary for the generation of an AHP. This is shown by our finding that a corresponding outward current is elicited by rectangular depolarization, which is not accompanied by 'spikelets' under voltage clamp, unlike the depolarization of VS and HS neurons during visual stimulation. In this respect the mechanism that produces an AHP in fly TCs differs from the spike-dependent mechanism in other cell types (see e.g. Sanchez-Vives et al., 2000).

\section{Functional implications of a control of after-hyperpolarization by membrane depolarization}

The results of the present study indicate that in fly TCs AHP and, thus, direction-selective adaptation that is assumed to be caused during motion stimulation preceding the AHP by the same mechanisms are regulated by depolarization of the dendrite. This kind of regulation makes a functionally relevant difference to a putative, alternative regulation by an input-driven but depolarization-independent process. The reason for this lies in the activation characteristics of synaptic inputs of TCs. These inputs have been concluded to be only weakly tuned to opposite directions of motion (Borst et al., 1995; Single et al., 1997). Thus, excitatory and inhibitory synaptic inputs of TCs are activated in conjunction when a stimulus moves in either the preferred direction or the antipreferred direction (see Fig. 6, inset). How far this input activity results in a net depolarization (during preferred-direction motion) or in a net hyperpolarization (during motion in the anti-preferred direction) of the TCs depends on the activation ratio between excitatory and inhibitory inputs. This activation ratio depends on the direction and velocity of motion (Egelhaaf \& Borst, 1989; Borst \& Egelhaaf, 1990). Thus, a control of adaptation by membrane depolarization, as demonstrated in the present study, provides a more direction-selective mechanism for the control of adaptation than putative alternative control mechanisms, in particular signaling pathways regulated by synaptic input. Similar considerations hold true for a putative regulation of adaptation by the cytosolic $\mathrm{Ca}^{2+}$ level. Although strong depolarization-activated $\mathrm{Ca}^{2+}$ influx has been demonstrated in TCs, a sizeable fraction of the $\mathrm{Ca}^{2+}$ accumulation during visual stimulation appears to be due to $\mathrm{Ca}^{2+}$ influx through acetylcholine receptors (Borst \& Single, 2000; Single \& Borst, 2002). Given the above considerations of the weak direction selectivity of TC inputs, a putative adaptation process that is regulated by the cytosolic level of $\mathrm{Ca}^{2+}$ or $\mathrm{Na}^{+}$would also be expected to be activated by motion in the anti-preferred direction. Moreover, it would be expected to be differentially pronounced at different stimulus velocities. This appears not to be the case for the AHP of fly TCs. In HS neurons of Eristalis tenax, the amplitude of the AHP was shown to be closely correlated with the depolarization induced by the adapting stimulus, regardless of whether the contrast, direction or velocity of the stimulus was varied to reach different depolarization levels (Harris et al., 2000).

It might be beneficial for a neuron when its adaptation state is regulated by depolarization rather than by other parameters. Adaptation has been proposed to prevent a neuron from operating in an activity range where its input/output function has only a shallow slope. The neuron would operate almost certainly in such an unfavorable operating range during strong depolarization. Thus, it is plausible that depolarization itself serves as an adapting signal that leads to a shift back to a less depolarized voltage level. Saving of energy has been suggested as a further function of adaptation (for review see Niven \& Laughlin, 2008). The ionic mechanisms underlying depolarization represent costly processes, again making it plausible to utilize depolarization as an adaptation signal.

Our results suggest that the cellular mechanism leading to AHP is located in the dendrite rather than in the axon. This localization might have important functional implications. On the one hand, if generated in the dendrite rather than in the axon, the hyperpolarization might exert a weaker impact on action potential generation. This aspect is, however, of minor relevance in the neurons analysed in the present study because they generate a predominantly graded output signal. On the other hand, if localized in the dendrite, the conductance change associated with the AHP might have a large impact on dendritic integration because it would have a shunting effect on currents generated by the synaptic inputs. The resulting adaptation effects might in this case even stay restricted to certain locations in the receptive field of the $\mathrm{TC}$, as their large dendrites receive synaptic input in a retinotopically organized manner. The AHP itself, even if generated by local visual stimulation, affects stimuli subsequently presented in a different location (Nordström \& O'Carroll, 2009). However, the putative decrease in gain associated with shunting of input currents would remain confined to a part of the dendrite and, thus, to stimuli located in a distinct region of the visual field.

\section{Acknowledgements}

This work was supported by a grant from the Deutsche Forschungsgemeinschaft (KU-1520/1). We are thankful to the anonymous reviewers for their helpful comments on previous versions of our manuscript.

\section{Abbreviations}

AHP, after-hyperpolarization; BAPTA, 1,2-bis(o-aminophenoxy)ethane-N,N, $\mathrm{N}^{\prime}, \mathrm{N}^{\prime}$-tetraacetic acid; $\mathrm{CH}$, centrifugal horizontal; HS, horizontal system; LED, light-emitting diode; TC, tangential cell; UV, ultraviolet; VS, vertical system. 


\section{References}

Arganda, S., Guantes, R. \& de Polavieja, G.G. (2007) Sodium pumps adapt spike bursting to stimulus statistics. Nat. Neurosci., 10, 1467-1473.

Bellone, C., Luscher, C. \& Mameli, M. (2008) Mechanisms of synaptic depression triggered by metabotropic glutamate receptors. Cell. Mol. Life Sci., 65, 2913-2923.

Bhattacharjee, A. \& Kaczmarek, L.K. (2005) For $\mathrm{K}^{+}$channels, $\mathrm{Na}^{+}$is the new $\mathrm{Ca}^{2+}$. Trends Neurosci., 28, 422-428.

Borst, A. \& Egelhaaf, M. (1987) Temporal modulation of luminance adapts time constant of fly movement detectors. Biol. Cybern., 56, 209-215.

Borst, A. \& Egelhaaf, M. (1989) Principles of visual motion detection. Trends Neurosci., 12, 297-306.

Borst, A. \& Egelhaaf, M. (1990) Direction selectivity of blowfly motionsensitive neurons is computed in a two-stage process. Proc. Natl Acad. Sci. USA, 87, 9363-9367.

Borst, A. \& Egelhaaf, M. (1992) In vivo imaging of calcium accumulation in fly interneurons as elicited by visual motion stimulation. Proc. Natl Acad. Sci. USA, 89, 4139-4143.

Borst, A. \& Haag, J. (1996) The intrinsic electrophysiological characteristics of fly lobula plate tangential cells: I. Passive membrane properties. J. Comput. Neurosci., 3, 313-336.

Borst, A. \& Haag, J. (2002) Neural networks in the cockpit of the fly. J. Comp. Physiol. [A], 188, 419-437.

Borst, A. \& Single, S. (2000) Local current spread in electrically compact neurons of the fly. Neurosci. Lett., 285, 123-126.

Borst, A., Egelhaaf, M. \& Haag, J. (1995) Mechanisms of dendritic integration underlying gain control in fly motion-sensitive interneurons. J. Comput. Neurosci., 2, 5-18.

Brenner, N., Bialek, W. \& de Ruyter van Steveninck, R.R. (2000) Adaptive rescaling maximizes information transmission. Neuron, 26, 695-702.

Dürr, V. \& Egelhaaf, M. (1999) In vivo calcium accumulation in presynaptic and postsynaptic dendrites of visual interneurons. J. Neurophysiol., 82, $3327-3338$.

Dutar, P., Petrozzino, J.J., Vu, H.M., Schmidt, M.F. \& Perkel, D.J. (2000) Slow synaptic inhibition mediated by metabotropic glutamate receptor activation of GIRK channels. J. Neurophysiol., 84, 2284-2290.

Eckert, H. \& Dvorak, D.R. (1983) The centrifugal horizontal cells in the lobula plate of the blowfly, Phaenicia sericata. J. Insect Physiol., 29, 547-560.

Egelhaaf, M. (2006) The neural computation of visual motion information. In Warrant, E. \& Nilsson, D.-E. (Eds), Invertebrate Vision. Cambridge University Press, Cambridge, pp. 399-461.

Egelhaaf, M. (2008) Fly vision: neural mechanisms of motion computation. Curr. Biol., 18, R339-R341.

Egelhaaf, M. \& Borst, A. (1989) Transient and steady-state response properties of movement detectors. J. Opt. Soc. Am. A, 6, 116-127.

Egelhaaf, M., Kern, R., Krapp, H.G., Kretzberg, J., Kurtz, R. \& Warzecha, A.K. (2002) Neural encoding of behaviourally relevant visual-motion information in the fly. Trends Neurosci., 25, 96-102.

Gerster, U., Stavenga, D.G. \& Backhaus, W. (1997) $\mathrm{Na}^{+} / \mathrm{K}^{+}$-pump activity in photoreceptors of the blowfly Calliphora: a model analysis based on membrane potential measurements. J. Comp. Physiol. [A], 180, 113-122.

Haag, J. \& Borst, A. (2000) Spatial distribution and characteristics of voltagegated calcium signals within visual interneurons. J. Neurophysiol., 83, 10391051.

Haag, J. \& Borst, A. (2002) Dendro-dendritic interactions between motionsensitive large-field neurons in the fly. J. Neurosci., 22, 3227-3233.

Haag, J., Theunissen, F. \& Borst, A. (1997) The intrinsic electrophysiological characteristics of fly lobula plate tangential cells: II. Active membrane properties. J. Comput. Neurosci., 4, 349-369.

Haag, J., Wertz, A. \& Borst, A. (2007) Integration of lobula plate output signals by DNOVS1, an identified premotor descending neuron. J. Neurosci., 27, 1992-2000.

Harris, R.A., O'Carroll, D.C. \& Laughlin, S.B. (1999) Adaptation and the temporal delay filter of fly motion detectors. Vision Res., 39, 2603-2613.

Harris, R.A., O'Carroll, D.C. \& Laughlin, S.B. (2000) Contrast gain reduction in fly motion adaptation. Neuron, 28, 595-606.

Hausen, K. (1982) Motion sensitive interneurons in the optomotor system of the fly: I. The horizontal cells: structure and signals. Biol. Cybern., 45, 143-156.

Hausen, K. (1984) The lobula-complex of the fly: structure, function and significance in visual behaviour. In Ali, M.A. (Ed.), Photoreception and Vision in Invertebrates. Plenum Press, New York, London, pp. 523-559.

Hausen, K. \& Egelhaaf, M. (1989) Neural mechanisms of visual course control in insects. In Stavenga, D.G. \& Hardie, R.C. (Eds), Facets of Vision. Springer, Berlin, Heidelberg, pp. 391-424.
Hawkins, R.D., Kandel, E.R. \& Bailey, C.H. (2006) Molecular mechanisms of memory storage in Aplysia. Biol. Bull., 210, 174-191.

Hengstenberg, R. (1977) Spike responses of 'non-spiking' visual interneurone. Nature, 270, 338-340.

Hengstenberg, R. (1982) Common visual response properties of giant vertical cells in the lobula plate of the blowfly Calliphora. J. Comp. Physiol. [A], 149, 179-193.

Huston, S.J. \& Krapp, H.G. (2008) Visuomotor transformation in the fly gaze stabilization system. PLoS Biol., 6, e173.

Jansen, J.K. \& Nicholls, J.G. (1973) Conductance changes, an electrogenic pump and the hyperpolarization of leech neurones following impulses. J. Physiol., 229, 635-655.

Jansonius, N.M. (1990) Properties of the sodium pump in the blowfly photoreceptor cell. J. Comp. Physiol. [A], 167, 461-467.

Kirschfeld, K. \& Franceschini, N. (1977) Evidence for a sensitising pigment in fly photoreceptors. Nature, 269, 386-390.

Knoflach, F. \& Kemp, J.A. (1998) Metabotropic glutamate group II receptors activate a $\mathrm{G}$ protein-coupled inwardly rectifying $\mathrm{K}^{+}$current in neurones of the rat cerebellum. J. Physiol., 509, 347-354.

Kohn, A. (2007) Visual adaptation: physiology, mechanisms, and functional benefits. J. Neurophysiol., 97, 3155-3164.

Kurtz, R. (2004) $\mathrm{Ca}^{2+}$ clearance in visual motion-sensitive neurons of the fly studied in vivo by sensory stimulation and UV photolysis of caged $\mathrm{Ca}^{2+}$. J. Neurophysiol., 92, 458-467.

Kurtz, R. (2007) Direction-selective adaptation in fly visual motion-sensitive neurons is generated by an intrinsic conductance-based mechanism. Neuroscience, 146, 573-583.

Kurtz, R., Dürr, V. \& Egelhaaf, M. (2000) Dendritic calcium accumulation associated with direction-selective adaptation in visual motion-sensitive neurons in vivo. J. Neurophysiol., 84, 1914-1923.

Kurtz, R., Warzecha, A.K. \& Egelhaaf, M. (2001) Transfer of visual motion information via graded synapses operates linearly in the natural activity range. J. Neurosci., 21, 6957-6966.

Laughlin, S.B. (1981) A simple coding procedure enhances a neuron's information capacity. Z. Naturforsch., 36c, 910-912.

Maddess, T. \& Laughlin, S.B. (1985) Adaptation of the motion-sensitive neuron $\mathrm{H} 1$ is generated locally and governed by contrast frequency. Proc. R. Soc. Lond. B Biol. Sci., 228, 251-275.

Manookin, M.B. \& Demb, J.B. (2006) Presynaptic mechanism for slow contrast adaptation in mammalian retinal ganglion cells. Neuron, 50, 453-464.

Neri, P. (2007) Fast-scale adaptive changes of directional tuning in fly tangential cells are explained by a static nonlinearity. J. Exp. Biol., 210, 3199-3208.

Niven, J.E. \& Laughlin, S.B. (2008) Energy limitation as a selective pressure on the evolution of sensory systems. J. Exp. Biol., 211, 1792-1804.

Nordström, K. \& O'Carroll, D.C. (2009) The motion after-effect: local and global contributions to contrast sensitivity. Proc. Biol. Sci., 276, 1545-1554.

Peron, S. \& Gabbiani, F. (2009) Spike frequency adaptation mediates looming stimulus selectivity in a collision-detecting neuron. Nat. Neurosci., 12, 318-326.

de Ruyter van Steveninck, R.R., Zaagman, W.H. \& Mastebroeck, H.A.K. (1986) Adaptation of transient responses of a movement-sensitive neuron in the visual system of the blowfly Calliphora erythrocephala. Biol. Cybern., 54, 223-236.

Sanchez-Vives, M.V., Nowak, L.G. \& McCormick, D.A. (2000) Cellular mechanisms of long-lasting adaptation in visual cortical neurons in vitro. J. Neurosci., 20, 4286-4299.

Single, S. \& Borst, A. (2002) Different mechanisms of calcium entry within different dendritic compartments. J. Neurophysiol., 87, 1616-1624.

Single, S., Haag, J. \& Borst, A. (1997) Dendritic computation of direction selectivity and gain control in visual interneurons. J. Neurosci., 17, 6023-6030.

Strausfeld, N.J. \& Bassemir, U.K. (1985) Lobula plate and ocellar interneurons converge onto a cluster of descending neurons leading to neck and leg motor neuropil in Calliphora erythrocephala. Cell Tissue Res., 240, 617-640.

Torkkeli, P.H., Sekizawa, S. \& French, A.S. (2001) Inactivation of voltageactivated $\mathrm{Na}(+)$ currents contributes to different adaptation properties of paired mechanosensory neurons. J. Neurophysiol., 85, 1595-1602.

Vähäsöyrinki, M., Niven, J.E., Hardie, R.C., Weckstrom, M. \& Juusola, M. (2006) Robustness of neural coding in Drosophila photoreceptors in the absence of slow delayed rectifier $\mathrm{K}^{+}$channels. J. Neurosci., 26, 2652-2660.

Wang, X.J. (1998) Calcium coding and adaptive temporal computation in cortical pyramidal neurons. J. Neurophysiol., 79, 1549-1566.

Warzecha, A.K., Kurtz, R. \& Egelhaaf, M. (2003) Synaptic transfer of dynamic motion information between identified neurons in the visual system of the blowfly. Neuroscience, 119, 1103-1112.

Wen, H., Weiger, T.M., Ferguson, T.S., Shahidullah, M., Scott, S.S. \& Levitan, I.B. (2005) A Drosophila KCNQ channel essential for early embryonic development. J. Neurosci., 25, 10147-10156. 